\title{
Existence of Solutions for Klein-Gordon-Maxwell Equations Involving Hardy-Sobolev Critical Exponents
}

\author{
Xin Zhang \\ School of Mathematics, Liaoning Normal University, Dalian, China \\ Email: Zhang_Xinbb@163.com
}

How to cite this paper: Zhang, X. (2021) Existence of Solutions for Klein-GordonMaxwell Equations Involving Hardy-Sobolev Critical Exponents. Open Access Library Journal, 8: e7709.

https://doi.org/10.4236/oalib.1107709

Received: June 28, 2021

Accepted: August 27, 2021

Published: August 30, 2021

Copyright $\odot 2021$ by author(s) and Open Access Library Inc.

This work is licensed under the Creative

Commons Attribution International

License (CC BY 4.0).

http://creativecommons.org/licenses/by/4.0/

\begin{abstract}
We investigate the existence of solutions for Klein-Gordon-Maxwell equations involving Hardy-Sobolev critical exponents. By means of the Ekeland's variational principle and the Mountain Pass Theorem, we obtain that there is at least a nontrivial solution for the subcritical system. Then we prove that there are at least two different solutions for the critical system.

\section{Subject Areas \\ Functional Analysis}

\section{Keywords}

Existence of Solutions, Klein-Gordon-Maxwell Equations, Hardy-Sobolev

Critical Exponents
\end{abstract}

\section{Introduction and Preliminaries}

In recent years, great attention has been given to problems driven by the Laplacian. One of the reasons for this comes from the fact that this operator appears in several applications in different subjects, such as flame propagation, free boundary obstacle problems, and ultrarelativistic limits of quantum mechanics. In particular, from a probabilistic point of view, the Laplace operator is the infinitesimal generator of a Lévy process. For more details and applications, see other references [1] [2].

Problems with two nonlinearities recently have been studied by several authors. In particular, such problems were considered in [3] [4] for the Laplacian, the $p$-Laplacian, the Biharmonic operator and the fractional Laplacian. In [5], Ghoussoub, Robert and Shakerian investigated problems with doubly critical 
nonlinear terms, with either critical Sobolev term or critical Hardy-Sobolev term, for the Laplacian and the fractional Laplacian.

Solutions of critical Sobolev problems were found in [6], as critical points of a suitable functional, by the Mountain-Pass lemma without the $(P S)_{c}$ condition. In this case, the $(P S)_{c}$ condition only holds true for $c$ in certain intervals related to the best Sobolev constant. In the control of the Mountain-Pass level, the extremal function of the best Sobolev constant plays an important role.

For example, Jannelli (see [7]) considered that the problem

$$
\left\{\begin{array}{l}
-\Delta u-\lambda u=\mu \frac{u}{|x|^{2}}+\frac{|u|^{2^{*}(s)-2}}{|x|^{s}} u, \quad x \in \Omega, \\
u=0, \quad x \in \partial \Omega,
\end{array}\right.
$$

they proved the existence of nontrivial solutions for the preceding equations involving a critical Hardy-Sobolev exponent in a bounded domain.

Then Kang and Peng in [8] considered the following problem based on the above equations

$$
\left\{\begin{array}{l}
-\Delta u-\mu \frac{u}{|x|^{2}}=\frac{u^{2^{*}(s)-2}}{|x|^{s}} u+\lambda|u|^{r-2} u, \quad x \in \Omega, \\
u=0, \quad x \in \partial \Omega .
\end{array}\right.
$$

They established the problem above has at least a pair of sign-changing solutions with $\lambda>0$ and $2<r<2^{*}$ in this reference [8]. Kang and Peng in [9] proved that problem above has at least one positive solution under some conditions for $r, \mu$ and $\lambda$. They also proved in [10] that as $r=2$, the problem above has at least a pair of sign-changing solutions for $N \geq 7, \mu \in[0, \bar{\mu}-4)$ and $0<\lambda<\lambda_{1}(u)$.

Motivated by the study of solitary waves of the nonlinear Klein-Gordon equation interacting with an electromagnetic field, Benci and Fortunato derived in [11] a model that is described by the following elliptic system

$$
\left\{\begin{array}{l}
-\Delta u+\left[m_{0}^{2}-(\omega+\phi)^{2}\right] u=|u|^{p-2} u, \\
\Delta \phi=(\omega+\phi) u^{2},
\end{array}\right.
$$

where $m_{0}$ and $\omega$ are real constants. They proved existence of infinitely many radially symmetric solutions $(u, \phi) \in H^{1}\left(\mathbb{R}^{3}\right) \times D^{1,2}\left(\mathbb{R}^{3}\right)$ for the above system when $\left|m_{0}\right|>|\omega|$ and for sub-critical exponents $p$ satisfying $4<p<2^{*}$. As in [12], they derived a variational identity to prove the non-existence of nontrivial weak solutions for the system above. In [13], Cassani investigated the critical case i.e. $p=2^{*}=\frac{2 N}{N-2}$, the critical Sobolev exponent. Moreover, Cassani used a Pohozaev-type argument, which points out an invariance property for the problem (3), to prove non-existence of solutions with a suitable decay at infinity and in particular it turns out to be the case of radially symmetric solutions. In [13], Cassani replaced the first equation of the System (3), adding a lower order per- 
turbation, by the following

$$
-\Delta u+\left[m_{0}^{2}-(\omega+\phi)^{2}\right] u=\mu|u|^{p-2} u+|u|^{2^{*}-2} u,
$$

where $\mu>0$ and $4 \leq p<6=2^{*}$. In this case, they recovered a Mountain-Pass type solution for Equation (4) and the second equation of System (3).

The above-mentioned equations with Hardy-Sobolev critical exponents are restricted to bounded regions, and the two Klein-Gordon-Maxwell systems mentioned above involve Sobolev critical exponents, not Hardy-Sobolev critical exponents. Moreover, some parts of the certification process did not give a specific certification process. We are inspired by the proof methods of a nontrivial solution and infinitely many solutions in the above-mentioned literatures, and investigate the existence of solutions for subcritical equations and critical equations with Hardy-Sobolev critical exponents in $\mathbb{R}^{3}$.

In this paper, firstly we study existence of solutions for the following KleinGordon-Maxwell equations involving Hardy-Sobolev critical exponents

$$
\begin{cases}-\Delta u+\left[m^{2}-(\omega+\phi)^{2}\right] u=\frac{|u|^{p-2} u}{|x|^{s}}, & x \in \mathbb{R}^{3} \\ -\Delta \phi+u^{2} \phi=-\omega u^{2}, & x \in \mathbb{R}^{3}\end{cases}
$$

where $m$ and $\omega$ are real constants, $2<p<2^{*}(s)=\frac{2(N-s)}{N-2}, 0<s<2$.

Then we study existence of solutions for the following equations

$$
\begin{cases}-\Delta u+\left[m^{2}-(\omega+\phi)^{2}\right] u=\mu \frac{|u|^{q-2} u}{|x|^{s}}+\frac{|u|^{p-2} u}{|x|^{s}}+\frac{|u|^{2^{*}(s)-2} u}{|x|^{s}}, & x \in \mathbb{R}^{3} \\ -\Delta \phi+u^{2} \phi=-\omega u^{2}, & x \in \mathbb{R}^{3}\end{cases}
$$

where $m, \mu$ and $\omega$ are real constants, $1<q<2,2<p<2^{*}(s)=\frac{2(N-s)}{N-2}$, $0<s<2$.

In Section 2, we first give main results for the Systems (5) and (6). In Section 3, we prove the existence of solutions of System (5). In Section 4, we establish existence of solutions of System (6).

\section{Main Results}

Throughout this paper, we denote the $L^{p}\left(\mathbb{R}^{3},|x|^{-s} \mathrm{~d} x\right)$ norm by

$$
|u|_{p, s}=\left(\int_{\mathbb{R}^{3}} \frac{|u|^{p}}{|x|^{s}} \mathrm{~d} x\right)^{\frac{1}{p}}
$$

and $L^{p}\left(\mathbb{R}^{3}, \mathrm{~d} x\right)$ norm by $|u|_{p}=\left(\int_{\mathbb{R}^{3}}|u|^{p} \mathrm{~d} x\right)^{\frac{1}{p}}$. For simplicity, set

$$
\begin{aligned}
& |u|_{2^{*}(0), 0}=|u|_{2^{*}}=|u|_{6}, \text { since } 2^{*}=\frac{2 N}{N-2} . \\
& D^{1,2}\left(\mathbb{R}^{3}\right)=\left\{u \in L^{2^{*}}\left(\mathbb{R}^{3}\right):|\nabla u| \in L^{2}\left(\mathbb{R}^{3}\right)\right\} \text { is a Sobolev space with norm }
\end{aligned}
$$




$$
\|u\|_{D^{1,2}}=\left(\int_{\mathbb{R}^{3}}|\nabla u|^{2} \mathrm{~d} x\right)^{\frac{1}{2}} .
$$

Moreover, $H^{1}\left(\mathbb{R}^{3}\right)$ is the usual Sobolev space with norm

$$
\|u\|_{H^{1}}=\left(\int_{\mathbb{R}^{3}}\left(|\nabla u|^{2}+u^{2}\right) \mathrm{d} x\right)^{\frac{1}{2}}
$$

continuously embedded in $L^{p}\left(\mathbb{R}^{3}\right)$ for $2 \leq p \leq 2^{*}(s)$. Define Hardy-Sobolev best constant as follows

$$
S_{s}=\inf _{u \in D^{1,2}\left(\mathbb{R}^{3}\right), u \neq 0} \frac{\int_{\mathbb{R}^{3}}|\nabla u|^{2} \mathrm{~d} x}{\left(\int_{\mathbb{R}^{3}} \frac{|u|^{2^{*}(s)}}{|x|^{s}} \mathrm{~d} x\right)^{\frac{2}{2^{*}(s)}}} .
$$

Theorem 2.1. If one of the following conditions is satisfied:

1) $2<p<4$ and $\left(\frac{p}{2}-1\right) m^{2}>\omega^{2}$, or

2) $4 \leq p<2^{*}(s)$ and $|m|>|\omega|$.

Then System (5) admits at least a nontrivial solution.

Theorem 2.2. If one of the following conditions is satisfied:

1) $4 \leq p<2^{*}(s)$ and $|m|>|\omega|$, or

2) $2<p<4$ and $\left(\frac{p}{2}-1\right) m^{2}>\omega^{2}$.

Then there exists a constant $\tilde{m}>0$ such that System (6) admits at least two different solutions $(u, \phi)$ satisfying $\|u\|_{H^{1}}<+\infty,\|\phi\|_{D^{1,2}}<+\infty \quad$ when $0<\mu<\tilde{m}$.

We define the functional of System (5)

$$
F(u, \phi)=\frac{1}{2} \int_{\mathbb{R}^{3}}\left(|\nabla u|^{2}-|\nabla \phi|^{2}+\left[m^{2}-(\omega+\phi)^{2}\right] u^{2}\right) \mathrm{d} x-\frac{1}{p} \int_{\mathbb{R}^{3}} \frac{|u|^{p}}{|x|^{s}} \mathrm{~d} x .
$$

Define the functional of System (6) as follows

$$
\begin{aligned}
\tilde{F}(u, \phi)= & \frac{1}{2} \int_{\mathbb{R}^{3}}\left(|\nabla u|^{2}-|\nabla \phi|^{2}+\left[m^{2}-(\omega+\phi)^{2}\right] u^{2}\right) \mathrm{d} x \\
& -\frac{\mu}{q} \int_{\mathbb{R}^{3}} \frac{|u|^{q}}{|x|^{s}} \mathrm{~d} x-\frac{1}{p} \int_{\mathbb{R}^{3}} \frac{|u|^{p}}{|x|^{s}} \mathrm{~d} x-\frac{1}{2^{*}(s)} \int_{\mathbb{R}^{3}} \frac{|u|^{2^{*}(s)}}{|x|^{s}} \mathrm{~d} x .
\end{aligned}
$$

Remark 2.1. The functional $F$ and $\tilde{F}$ are strongly indefinite i.e. unbounded from below and from above on infinite dimensional subspaces. In order to avoid this indefiniteness, which rules out many of the usual tools of critical point theory, a reduction method is performed in [11] which we now recall. For $u$ and $\phi$ defined above, we have the following lemmas.

\section{The Proof of Theorem 2.1}

Lemma 3.1. For every $u \in H^{1}\left(\mathbb{R}^{3}\right)$,

1) there exists a unique function $\phi=\Phi(u) \in D^{1,2}\left(\mathbb{R}^{3}\right)$ that solves the second equation of System (5); 
2) if $u$ is radially symmetric, then $\Phi(u)$ is radial too;

3) $\Phi(u)(x) \leq 0$, moreover, $\Phi(u)(x) \geq-\omega$, if $u(x) \neq 0$ and $\omega>0$.

Proof. The first result is proved in Lemma 3 of [14]. While the second one, though not explicitly stated, is proved in Lemma 5 of [14]. The third result can be found in Lemma 2.3 of [15].

Lemma 3.2. The map $\Phi$ is $C^{1}$ and $G_{\phi}=\left\{(u, \phi) \in H^{1}\left(\mathbb{R}^{3}\right) \times D^{1,2}\left(\mathbb{R}^{3}\right) \mid F_{\phi}^{\prime}(u, \phi)=0\right\}$.

Proof. Noticing that $\Phi(u)$ is a solution of the second equation in System (5), we have

$$
-\int_{\mathbb{R}^{3}}|\nabla \Phi(u)|^{2} \mathrm{~d} x=\int_{\mathbb{R}^{3}} \omega \Phi(u) u^{2} \mathrm{~d} x+\int_{\mathbb{R}^{3}} \Phi^{2}(u) u^{2} \mathrm{~d} x .
$$

In addition,

$$
\begin{aligned}
F(u, \Phi(u))= & \frac{1}{2} \int_{\mathbb{R}^{3}}\left(|\nabla u|^{2}-|\nabla \Phi(u)|^{2}+\left[m^{2}-(\omega+\Phi(u))^{2}\right] u^{2}\right) \mathrm{d} x \\
& -\frac{1}{p} \int_{\mathbb{R}^{3}} \frac{|u|^{p}}{|x|^{s}} \mathrm{~d} x, \\
F_{\phi}^{\prime}(u, \Phi(u))= & -\int_{\mathbb{R}^{3}}|\nabla \Phi(u)|^{2} \mathrm{~d} x-\int_{\mathbb{R}^{3}} \omega \Phi(u) u^{2} \mathrm{~d} x-\int_{\mathbb{R}^{3}} \Phi^{2}(u) u^{2} \mathrm{~d} x .
\end{aligned}
$$

According to (9), one gets $F_{\phi}^{\prime}(u, \Phi(u))=0$ for any

$(u, \phi) \in H^{1}\left(\mathbb{R}^{3}\right) \times D^{1,2}\left(\mathbb{R}^{3}\right)$. Thus

$F^{\prime}(u, \Phi(u))=F_{u}^{\prime}(u, \Phi(u))+F_{\phi}^{\prime}(u, \Phi(u)) \Phi^{\prime}(u)=F_{u}^{\prime}(u, \Phi(u))$.

Define $I(u)=F(u, \phi)$. If $u, v \in H^{1}\left(\mathbb{R}^{3}\right)$, then one has

$$
I^{\prime}(u) v=\int_{\mathbb{R}^{3}}\left(\nabla u \cdot \nabla v+\left[m^{2}-(\omega+\phi)^{2}\right] u v\right) \mathrm{d} x-\int_{\mathbb{R}^{3}} \frac{|u|^{p-2}}{|x|^{s}} u v \mathrm{~d} x .
$$

\section{Lemma 3.3. The following statements are equivalent.}

1) $(u, \phi) \in H^{1}\left(\mathbb{R}^{3}\right) \times D^{1,2}\left(\mathbb{R}^{3}\right)$ is a solution of System (5);

2) $u$ is a critical point for $I$ and $\phi=\Phi(u)$.

Proof. 2) $\Rightarrow$ 1) Obviously.

1) $\Rightarrow 2$ ) Suppose $F_{u}^{\prime}(u, \phi)$ and $F_{\phi}^{\prime}(u, \phi)$ denote the partial derivatives of $F$ at $(u, \phi) \in H^{1}\left(\mathbb{R}^{3}\right) \times D^{1,2}\left(\mathbb{R}^{3}\right)$. Then for every $v \in H^{1}\left(\mathbb{R}^{3}\right)$ and $\psi \in D^{1,2}\left(\mathbb{R}^{3}\right)$, one gets

$$
\begin{gathered}
F_{u}^{\prime}(u, \phi)[v]=\int_{\mathbb{R}^{3}}\left(\nabla u \cdot \nabla v+\left[m^{2}-(\omega+\phi)^{2}\right] u v\right) \mathrm{d} x-\int_{\mathbb{R}^{3}} \frac{|u|^{p-2}}{|x|^{s}} u v \mathrm{~d} x, \\
F_{\phi}^{\prime}(u, \phi)[\psi]=-\int_{\mathbb{R}^{3}} \nabla \phi \cdot \nabla \psi \mathrm{d} x-\int_{\mathbb{R}^{3}}(\omega+\phi) \psi u^{2} \mathrm{~d} x .
\end{gathered}
$$

By the standard computations, we can prove that $F_{u}^{\prime}(u, \phi)$ and $F_{\phi}^{\prime}(u, \phi)$ are continuous. From (11) and (12), it is easy to obtain that its critical points are solutions of System (5), by 1) of Lemma 3.1, one has $\phi=\Phi(u)$.

Lemma 3.4. For $u \in H^{1}\left(\mathbb{R}^{3}\right)$, if $|m|>|\omega|$, then there exist some constants $\rho_{1}, \alpha_{1}>0$ such that $\left.I(u)\right|_{\|\|_{H^{1}}=\rho_{1}} \geq \alpha_{1}>0$. 
Proof. From (7), one obtains

$$
\begin{aligned}
I(u)= & \frac{1}{2} \int_{\mathbb{R}^{3}}\left(|\nabla u|^{2}+\left(m^{2}-\omega^{2}\right) u^{2}\right) \mathrm{d} x-\frac{1}{2} \int_{\mathbb{R}^{3}}|\nabla \phi|^{2} \mathrm{~d} x \\
& -\int_{\mathbb{R}^{3}} \omega \phi u^{2} \mathrm{~d} x-\frac{1}{2} \int_{\mathbb{R}^{3}} \phi^{2} u^{2} \mathrm{~d} x-\frac{1}{p} \int_{\mathbb{R}^{3}} \frac{|u|^{p}}{|x|^{s}} \mathrm{~d} x .
\end{aligned}
$$

Substituting (9) into (13), we have

$$
\begin{aligned}
I(u)= & \frac{1}{2} \int_{\mathbb{R}^{3}}\left(|\nabla u|^{2}+\left(m^{2}-\omega^{2}\right) u^{2}\right) \mathrm{d} x+\frac{1}{2} \int_{\mathbb{R}^{3}}|\nabla \phi|^{2} \mathrm{~d} x \\
& +\frac{1}{2} \int_{\mathbb{R}^{3}} \phi^{2} u^{2} \mathrm{~d} x-\frac{1}{p} \int_{\mathbb{R}^{3}} \frac{|u|^{p}}{|x|^{s}} \mathrm{~d} x \\
\geq & \frac{\min \left\{1, m^{2}-\omega^{2}\right\}}{2} \int_{\mathbb{R}^{3}}\left(|\nabla u|^{2}+u^{2}\right) \mathrm{d} x-\frac{1}{p} \int_{\mathbb{R}^{3}} \frac{|u|^{p}}{|x|^{s}} \mathrm{~d} x \\
\geq & C_{1}\|u\|_{H^{1}}^{2}-C_{2}\|u\|_{H^{1}}^{p} \geq \alpha_{1}, \text { for } u \in H^{1}\left(\mathbb{R}^{3}\right),\|u\|_{H^{1}}=\rho_{1} .
\end{aligned}
$$

Thus

$$
\left.I(u)\right|_{\|u\|_{H^{1}}=\rho_{1}} \geq \alpha_{1}>0
$$

and the proof is completed.

Lemma 3.5. Under the assumptions of Theorem 2.1, there exists a function $\eta_{1} \in H^{1}\left(\mathbb{R}^{3}\right)$ with $\left\|\eta_{1}\right\|_{H^{1}}>\rho_{1}$ such that $I\left(\eta_{1}\right)<0$.

Proof. It is easy to obtain

$$
\begin{aligned}
\lim _{t \rightarrow+\infty} I(t u)= & \frac{t^{2}}{2} \int_{\mathbb{R}^{3}}\left(|\nabla u|^{2}+\left(m^{2}-\omega^{2}\right) u^{2}\right) \mathrm{d} x-t^{2} \int_{\mathbb{R}^{3}} \omega \Phi(t u) u^{2} \mathrm{~d} x \\
& -\frac{t^{2}}{2} \int_{\mathbb{R}^{3}} \Phi^{2}(t u) u^{2} \mathrm{~d} x-\frac{1}{2} \int_{\mathbb{R}^{3}}|\nabla \Phi(t u)|^{2} \mathrm{~d} x-\frac{t^{p}}{p} \int_{\mathbb{R}^{3}} \frac{|u|^{p}}{|x|^{s}} \mathrm{~d} x \\
\leq & \frac{t^{2}}{2} \int_{\mathbb{R}^{3}}\left(|\nabla u|^{2}+\left(m^{2}-\omega^{2}\right) u^{2}\right) \mathrm{d} x-\omega t^{2} \int_{\mathbb{R}^{3}} \Phi(t u) u^{2} \mathrm{~d} x-\frac{t^{p}}{p} \int_{\mathbb{R}^{3}} \frac{|u|^{p}}{|x|^{s}} \mathrm{~d} x \\
\leq & \frac{t^{2}}{2} \int_{\mathbb{R}^{3}}\left(|\nabla u|^{2}+\left(m^{2}-\omega^{2}\right) u^{2}+2 \omega^{2} u^{2}\right) \mathrm{d} x-\frac{t^{p}}{p} \int_{\mathbb{R}^{3}} \frac{|u|^{p}}{|x|^{s}} \mathrm{~d} x \\
\rightarrow & -\infty
\end{aligned}
$$

which implies that $I(u) \rightarrow-\infty$, as $\|u\|_{H^{1}} \rightarrow \infty$.

The lemma is proved by taking $\eta_{1}=t u$ with $t>0$ large enough and $u \neq 0$. Therefore we know that there exists $\eta_{1} \in H^{1}\left(\mathbb{R}^{3}\right),\left\|\eta_{1}\right\|_{H^{1}}>\rho_{1}$ such that $I\left(\eta_{1}\right)<0$.

Therefore, there exists a sequence $\left\{u_{n}\right\} \in H^{1}\left(\mathbb{R}^{3}\right)$, so-called Palais-Smale sequence, such that

$$
I\left(u_{n}\right) \rightarrow c>0 \text {, and } I^{\prime}\left(u_{n}\right) \rightarrow 0, \quad n \rightarrow \infty \text {, }
$$

where

$$
c=\inf _{\gamma \in \Gamma} \max _{t \in[0,1]} I(\gamma(t))
$$


with

$$
\Gamma=\left\{\gamma \in C\left([0,1], H^{1}\left(\mathbb{R}^{3}\right)\right): \gamma(0)=0, \gamma(1)=\eta_{1}\right\} .
$$

Since System (5) is set on $\mathbb{R}^{3}$, it is well known that the Sobolev embedding $H^{1}\left(\mathbb{R}^{3}\right) \hookrightarrow L^{p}\left(\mathbb{R}^{3},|x|^{-s} \mathrm{~d} x\right)\left(2 \leq p \leq 2^{*}(s)\right)$ is not compact and then it is usually difficult to prove that a Palais-Smale sequence is strongly convergent when we seek solutions of System (5) by variational methods. A standard tool to overcome the problem is to restrict ourselves to radial functions, namely we look at the functional $I$ on the subspace $H_{r}^{1}\left(\mathbb{R}^{3}\right)=\left\{u \in H^{1}\left(\mathbb{R}^{3}\right) \mid u(x)=u(|x|)\right\}$ and $D_{r}^{1,2}\left(\mathbb{R}^{3}\right)=\left\{u \in D^{1,2}\left(\mathbb{R}^{3}\right) \mid u(x)=u(|x|)\right\} \quad$ compactly embedded in $L_{r}^{p}\left(\mathbb{R}^{3},|x|^{-s} \mathrm{~d} x\right)$ for $2<p<2^{*}(s)$ and $L_{r}^{p}\left(\mathbb{R}^{3}, \mathrm{~d} x\right)$ for $2<p<2^{*}=\frac{2 N}{N-2}=6$. By standard arguments, one sees that if a critical point $u \in H_{r}^{1}\left(\mathbb{R}^{3}\right)$ for the functional $\left.I\right|_{H_{r}^{1}\left(\mathbb{R}^{3}\right)}$ is also a critical point of $I$.

Lemma 3.6. The PS sequence $\left\{u_{n}\right\} \in H_{r}^{1}\left(\mathbb{R}^{3}\right)$ given in (14) is bounded. Moreover, $\Phi\left(u_{n}\right) \in D_{r}^{1,2}\left(\mathbb{R}^{3}\right)$ is bounded, too.

Proof. Case 1. $2<p<4$. There exists a constant $C_{3}>0$, then by (7), (9) and (10), we get

$$
\begin{aligned}
& C_{3}+o(1)\left\|u_{n}\right\|_{H_{r}^{1}} \\
\geq & p I\left(u_{n}\right)-\left\langle I^{\prime}\left(u_{n}\right), u_{n}\right\rangle \\
= & \left(\frac{p}{2}-1\right) \int_{\mathbb{R}^{3}}\left(\left|\nabla u_{n}\right|^{2}+\left(m^{2}-\omega^{2}\right) u_{n}^{2}\right) \mathrm{d} x-\left(\frac{p}{2}-2\right) \int_{\mathbb{R}^{3}} \omega \Phi\left(u_{n}\right) u_{n}^{2} \mathrm{~d} x \\
& +\int_{\mathbb{R}^{3}} \Phi^{2}\left(u_{n}\right) u_{n}^{2} \mathrm{~d} x \\
\geq & \left(\frac{p}{2}-1\right) \int_{\mathbb{R}^{3}}\left|\nabla u_{n}\right|^{2} \mathrm{~d} x+\int_{\mathbb{R}^{3}}\left[\left(\frac{p}{2}-1\right)\left(m^{2}-\omega^{2}\right)+\left(\frac{p}{2}-2\right) \omega^{2}\right] u_{n}^{2} \mathrm{~d} x \\
\geq & C_{4}\left\|u_{n}\right\|_{H_{r}^{1}}^{2}
\end{aligned}
$$

for $n$ large enough. Therefore, it follows that $\left\{u_{n}\right\}$ is bounded in $H_{r}^{1}\left(\mathbb{R}^{3}\right)$.

Case 2. $4 \leq p<2^{*}(s)$. We have that there is a positive constant $\tilde{C}_{3}$ such that

$$
\tilde{C}_{3}+\left\|u_{n}\right\|_{H_{r}^{1}} \geq I\left(u_{n}\right)-\frac{1}{p}\left\langle I^{\prime}\left(u_{n}\right), u_{n}\right\rangle .
$$

According to (9), (10) and (13), $\Phi\left(u_{n}\right) \geq-\omega$, one has

$$
\begin{aligned}
\tilde{C}_{3} & +\left\|u_{n}\right\|_{H_{r}^{1}} \\
\geq & I\left(u_{n}\right)-\frac{1}{p}\left\langle I^{\prime}\left(u_{n}\right), u_{n}\right\rangle \\
= & \left(\frac{1}{2}-\frac{1}{p}\right) \int_{\mathbb{R}^{3}}\left(\left|\nabla u_{n}\right|^{2}+\left(m^{2}-\omega^{2}\right) u_{n}^{2}\right) \mathrm{d} x+\left(\frac{1}{2}+\frac{1}{p}\right) \int_{\mathbb{R}^{3}} \Phi^{2}\left(u_{n}\right) u_{n}^{2} \mathrm{~d} x \\
& +\frac{1}{2} \int_{\mathbb{R}^{3}}\left|\nabla \Phi\left(u_{n}\right)\right|^{2} \mathrm{~d} x+\frac{2}{p} \int_{\mathbb{R}^{3}} \omega \Phi\left(u_{n}\right) u_{n}^{2} \mathrm{~d} x
\end{aligned}
$$




$$
\begin{aligned}
= & \left(\frac{1}{2}-\frac{1}{p}\right) \int_{\mathbb{R}^{3}}\left(\left|\nabla u_{n}\right|^{2}+\left(m^{2}-\omega^{2}\right) u_{n}^{2}\right) \mathrm{d} x+\left(\frac{1}{2}-\frac{1}{p}\right) \int_{\mathbb{R}^{3}} \Phi^{2}\left(u_{n}\right) u_{n}^{2} \mathrm{~d} x \\
& +\left(\frac{1}{2}-\frac{2}{p}\right) \int_{\mathbb{R}^{3}}\left|\nabla \Phi\left(u_{n}\right)\right|^{2} \mathrm{~d} x \\
\geq & C_{5}\left\|u_{n}\right\|_{H_{r}^{1}}^{2}
\end{aligned}
$$

for $n$ large enough. It follows that $\left\{u_{n}\right\}$ is bounded in $H_{r}^{1}\left(\mathbb{R}^{3}\right)$.

According to Equation (9), one has

$$
\int_{\mathbb{R}^{3}}\left|\nabla \Phi\left(u_{n}\right)\right|^{2} \mathrm{~d} x=-\int_{\mathbb{R}^{3}} \omega \Phi\left(u_{n}\right) u_{n}^{2} \mathrm{~d} x-\int_{\mathbb{R}^{3}} \Phi^{2}\left(u_{n}\right) u_{n}^{2} \mathrm{~d} x,
$$

then by Hölder inequality

$$
\begin{aligned}
& \int_{\mathbb{R}^{3}}|f(x) \cdot g(x)| \mathrm{d} x \leq\left(\int_{\mathbb{R}^{3}}|f(x)|^{p} \mathrm{~d} x\right)^{\frac{1}{p}} \cdot\left(\int_{\mathbb{R}^{3}}|g(x)|^{q} \mathrm{~d} x\right)^{\frac{1}{q}} \\
& =|f(x)|_{p} \cdot|g(x)|_{q}, \quad\left(p>1, q>1, \frac{1}{p}+\frac{1}{q}=1\right)
\end{aligned}
$$

and Sobolev inequality

$$
|u|_{q} \leq C\|u\|_{H_{r}^{1}}
$$

One obtains

$$
\left\|\Phi\left(u_{n}\right)\right\|_{D_{r}^{1,2}}^{2} \leq C_{6}\left\|\Phi\left(u_{n}\right)\right\|_{D_{r}^{1,2}}\left\|u_{n}\right\|_{H_{r}^{1}}^{2} .
$$

Thus $\left\{\Phi\left(u_{n}\right)\right\}$ is bounded in $D_{r}^{1,2}\left(\mathbb{R}^{3}\right)$ by the boundedness of $\left\{u_{n}\right\} \subset H_{r}^{1}\left(\mathbb{R}^{3}\right)$.

Up to subsequence, we may assume that there exists $u \in H_{r}^{1}\left(\mathbb{R}^{3}\right)$ and $\varphi \in D_{r}^{1,2}\left(\mathbb{R}^{3}\right)$ such that

$$
\begin{gathered}
u_{n} \rightarrow u \quad \text { in } H_{r}^{1}\left(\mathbb{R}^{3}\right), \\
u_{n} \rightarrow u \quad \text { in } L_{r}^{p}\left(\mathbb{R}^{3}, \mathrm{~d} x\right) \text { for } 2<p<6, \\
\Phi\left(u_{n}\right) \rightarrow \varphi \text { in } D_{r}^{1,2}\left(\mathbb{R}^{3}\right) .
\end{gathered}
$$

Lemma 3.7. $\varphi=\Phi(u)$ and $\Phi\left(u_{n}\right) \rightarrow \Phi(u)$ in $D_{r}^{1,2}\left(\mathbb{R}^{3}\right)$.

Proof. First we prove the uniqueness. For every fixed $u \in H_{r}^{1}\left(\mathbb{R}^{3}\right)$, we consider the following minimizing problem $\inf _{\phi \in D_{r}^{1,2}\left(\mathbb{R}^{3}\right)} E_{u}(\phi)$, where $E_{u}: D_{r}^{1,2}\left(\mathbb{R}^{3}\right) \rightarrow \mathbb{R}$ defined as energy functional of the second equation in System (5).

$$
E_{u}(\phi)=\frac{1}{2} \int_{\mathbb{R}^{3}}|\nabla \phi|^{2} \mathrm{~d} x+\frac{1}{2} \int_{\mathbb{R}^{3}} \phi^{2} u^{2} \mathrm{~d} x+\int_{\mathbb{R}^{3}} \omega \phi u^{2} \mathrm{~d} x .
$$

In fact, by the proof of Lemma 2.1 in [16], one can know

$$
\Phi\left(u_{n}\right) \rightarrow \varphi \text {, locally uniformly in } \mathbb{R}^{3},
$$

so we obtain

$$
\int_{\mathbb{R}^{3}} \Phi\left(u_{n}\right) u_{n}^{2} \mathrm{~d} x \rightarrow \int_{\mathbb{R}^{3}} \varphi u^{2} \mathrm{~d} x, \quad \int_{\mathbb{R}^{3}} \Phi^{2}\left(u_{n}\right) u_{n}^{2} \mathrm{~d} x \rightarrow \int_{\mathbb{R}^{3}} \varphi^{2} u^{2} \mathrm{~d} x .
$$

From the weak lower semicontinuity of the norm in $D_{r}^{1,2}\left(\mathbb{R}^{3}\right)$ and the convergence above, one has 


$$
E_{u}(\varphi) \leq \liminf _{n \rightarrow \infty} E_{u_{n}}\left(\Phi\left(u_{n}\right)\right) \leq \liminf _{n \rightarrow \infty} E_{u_{n}}(\Phi(u))=E_{u}(\Phi(u)),
$$

then by 1 ) of Lemma 3.1, $\varphi=\Phi(u)$.

Next, we prove that $\left\{\Phi\left(u_{n}\right)\right\}$ converges strongly in $D_{r}^{1,2}\left(\mathbb{R}^{3}\right)$. Since $\Phi\left(u_{n}\right)$ satisfies the following equation

$$
\int_{\mathbb{R}^{3}} \nabla \Phi\left(u_{n}\right) \nabla \psi \mathrm{d} x+\int_{\mathbb{R}^{3}} u_{n}^{2} \Phi\left(u_{n}\right) \psi \mathrm{d} x=-\omega \int_{\mathbb{R}^{3}} u_{n}^{2} \psi \mathrm{d} x, \quad \psi \in D_{r}^{1,2}\left(\mathbb{R}^{3}\right) .
$$

Let us take the difference between (18) and the corresponding equation for $\Phi(u)$ to have

$$
\begin{aligned}
& \int_{\mathbb{R}^{3}} \nabla\left(\Phi\left(u_{n}\right)-\Phi(u)\right) \nabla \psi+u_{n}^{2}\left(\Phi\left(u_{n}\right)-\Phi(u)\right) \psi+\left(u_{n}^{2}-u^{2}\right) \Phi(u) \psi \mathrm{d} x \\
& =-\omega \int_{\mathbb{R}^{3}}\left(u_{n}^{2}-u^{2}\right) \psi \mathrm{d} x, \quad \psi \in D_{r}^{1,2}\left(\mathbb{R}^{3}\right) .
\end{aligned}
$$

Testing with $\psi=\left(\Phi\left(u_{n}\right)-\Phi(u)\right)$, by the Hölder inequality, the following holds

$$
\begin{aligned}
& \left\|\Phi\left(u_{n}\right)-\Phi(u)\right\|_{D_{r}^{1,2}}^{2} \\
& \leq|\omega| \int_{\mathbb{R}^{3}}\left|u_{n}^{2}-u^{2} \| \Phi\left(u_{n}\right)-\Phi(u)\right| \mathrm{d} x+\int_{\mathbb{R}^{3}}\left|u_{n}^{2}-u^{2}\right||\Phi(u)|\left|\Phi\left(u_{n}\right)-\Phi(u)\right| \mathrm{d} x \\
& \leq|\omega||| \Phi\left(u_{n}\right)-\left.\Phi(u)\right|_{6}\left|u_{n}^{2}-u^{2}\right|_{\frac{6}{5}}+|\Phi(u)|_{6}\left|\Phi\left(u_{n}\right)-\Phi(u)\right|_{6}\left|u_{n}^{2}-u^{2}\right|_{\frac{3}{2}} \\
& \leq C_{7}\left|u_{n}-u\right|_{\frac{12}{5}}+C_{8}\left|u_{n}-u\right|_{3},
\end{aligned}
$$

according to (16), one has $\Phi\left(u_{n}\right) \rightarrow \Phi(u)$ strongly in $D_{r}^{1,2}\left(\mathbb{R}^{3}\right)$.

Lemma 3.8. $\left\{u_{n}\right\} \in H_{r}^{1}\left(\mathbb{R}^{3}\right)$ has a strongly convergent subsequence in $H_{r}^{1}\left(\mathbb{R}^{3}\right)$.

Proof. Consider a sequence $\left\{u_{n}\right\}$ in $H_{r}^{1}\left(\mathbb{R}^{3}\right)$, which satisfies $I\left(u_{n}\right) \rightarrow c$, $I^{\prime}\left(u_{n}\right) \rightarrow 0$, and $\sup \left\|u_{n}\right\|_{H_{r}^{1}}<+\infty$. Going if necessary to a subsequence, since the embedding $H_{r}^{1}\left(\mathbb{R}^{3}\right) \hookrightarrow L_{r}^{p}\left(\mathbb{R}^{3},|x|^{-s} \mathrm{~d} x\right)$ is compact for any $p \in\left(2,2^{*}(s)\right)$, we have

$$
u_{n} \rightarrow u \text { in } L_{r}^{p}\left(\mathbb{R}^{3},|x|^{-s} \mathrm{~d} x\right) .
$$

According to (10), one obtains

$$
\begin{aligned}
& I^{\prime}\left(u_{n}\right)\left(u_{n}-u\right) \\
& =\int_{\mathbb{R}^{3}}\left(\nabla u_{n} \cdot \nabla\left(u_{n}-u\right)+\left[m^{2}-\left(\omega+\Phi\left(u_{n}\right)\right)^{2}\right] u_{n}\left(u_{n}-u\right)\right) \mathrm{d} x \\
& -\int_{\mathbb{R}^{3}} \frac{\left|u_{n}\right|^{p-2}}{|x|^{5}} u_{n}\left(u_{n}-u\right) \mathrm{d} x .
\end{aligned}
$$

Similarly, one gets

$$
\begin{aligned}
& I^{\prime}(u)\left(u_{n}-u\right) \\
& =\int_{\mathbb{R}^{3}}\left(\nabla u \cdot \nabla\left(u_{n}-u\right)+\left[m^{2}-(\omega+\Phi(u))^{2}\right] u\left(u_{n}-u\right)\right) \mathrm{d} x \\
& -\int_{\mathbb{R}^{3}} \frac{|u|^{p-2}}{|x|^{\mathrm{s}}} u\left(u_{n}-u\right) \mathrm{d} x .
\end{aligned}
$$

By (10), we easily get that 


$$
\begin{aligned}
& \int_{\mathbb{R}^{3}}\left(\left|\nabla\left(u_{n}-u\right)\right|^{2}+\left(m^{2}-\omega^{2}\right)\left(u_{n}-u\right)^{2}\right) \mathrm{d} x \\
& =\left\langle I^{\prime}\left(u_{n}\right)-I^{\prime}(u), u_{n}-u\right\rangle+\int_{\mathbb{R}^{3}}\left(\Phi^{2}\left(u_{n}\right) u_{n}-\Phi^{2}(u) u\right)\left(u_{n}-u\right) \mathrm{d} x \\
& \quad+\int_{\mathbb{R}^{3}}\left(\frac{\left|u_{n}\right|^{p-2}}{|x|^{s}} u_{n}-\frac{|u|^{p-2}}{|x|^{s}} u\right)\left(u_{n}-u\right) \mathrm{d} x \\
& \quad+2 \omega \int_{\mathbb{R}^{3}}\left(\Phi\left(u_{n}\right) u_{n}-\Phi(u) u\right)\left(u_{n}-u\right) \mathrm{d} x .
\end{aligned}
$$

It is clear that

$$
\left\langle I^{\prime}\left(u_{n}\right)-I^{\prime}(u), u_{n}-u\right\rangle \rightarrow 0 \text {, as } n \rightarrow \infty .
$$

Furthermore, in view of (21), we have

$$
\begin{aligned}
\int_{\mathbb{R}^{3}} \frac{\left|u_{n}\right|^{p-1}}{|x|^{s}}\left(u_{n}-u\right) \mathrm{d} x & \leq\left(\int_{\mathbb{R}^{3}} \frac{\left|u_{n}\right|^{p}}{|x|^{s}} \mathrm{~d} x\right)^{\frac{p-1}{p}}\left(\int_{\mathbb{R}^{3}} \frac{\left|u_{n}-u\right|^{p}}{|x|^{s}} \mathrm{~d} x\right)^{\frac{1}{p}} \\
& =\left|u_{n}\right|_{p, s}^{p-1}\left|u_{n}-u\right|_{p, s} \rightarrow 0, \text { as } n \rightarrow \infty .
\end{aligned}
$$

Similarly, we also obtain that

$$
\int_{\mathbb{R}^{3}} \frac{|u|^{p-1}}{|x|^{s}}\left(u_{n}-u\right) \mathrm{d} x \rightarrow 0, \text { as } n \rightarrow \infty .
$$

Thus combining (24) and (25), one gets that

$$
\begin{aligned}
& \int_{\mathbb{R}^{3}}\left(\frac{\left|u_{n}\right|^{p-2}}{|x|^{s}} u_{n}-\frac{|u|^{p-2}}{|x|^{s}} u\right)\left(u_{n}-u\right) \mathrm{d} x \\
& =\int_{\mathbb{R}^{3}} \frac{\left|u_{n}\right|^{p-1}}{|x|^{s}}\left(u_{n}-u\right) \mathrm{d} x-\int_{\mathbb{R}^{3}} \frac{|u|^{p-1}}{|x|^{s}}\left(u_{n}-u\right) \mathrm{d} x \\
& \rightarrow 0, \text { as } n \rightarrow \infty .
\end{aligned}
$$

By Hölder inequality and Sobolev inequality, one has

$$
\begin{aligned}
& \left|\int_{\mathbb{R}^{3}}\left(\Phi\left(u_{n}\right)-\Phi(u)\right) u_{n}\left(u_{n}-u\right) \mathrm{d} x\right| \\
& \leq\left|\left(\Phi\left(u_{n}\right)-\Phi(u)\right)\left(u_{n}-u\right)\right|_{2}\left|u_{n}\right|_{2} \\
& \leq\left|\Phi\left(u_{n}\right)-\Phi(u)\right|_{6}\left|u_{n}-u\right|_{3}\left|u_{n}\right|_{2} \\
& \leq C_{9}|| \Phi\left(u_{n}\right)-\left.\Phi(u)\right|_{D_{r^{1,2}}}\left|u_{n}-u\right|_{3}|| u_{n} \|_{H_{r}^{1}} .
\end{aligned}
$$

According to (16), one gets $\int_{\mathbb{R}^{3}}\left(\Phi\left(u_{n}\right)-\Phi(u)\right) u_{n}\left(u_{n}-u\right) \mathrm{d} x \rightarrow 0$, as $n \rightarrow \infty$. And

$$
\begin{aligned}
& \left|\int_{\mathbb{R}^{3}} \Phi(u)\left(u_{n}-u\right)^{2} \mathrm{~d} x\right| \leq|\Phi(u)|_{6}\left|u_{n}-u\right|_{3}\left|u_{n}-u\right|_{2} \\
& \leq C_{10}\|\Phi(u)\|_{D_{D^{1,2}}^{12}}\left|u_{n}-u\right|_{3}\left\|u_{n}-u\right\|_{H_{r}^{1}} \rightarrow 0, \text { as } n \rightarrow \infty .
\end{aligned}
$$

Thus we get that

$$
\begin{aligned}
& \int_{\mathbb{R}^{3}}\left(\Phi\left(u_{n}\right) u_{n}-\Phi(u) u\right)\left(u_{n}-u\right) \mathrm{d} x \\
& =\int_{\mathbb{R}^{3}}\left(\Phi\left(u_{n}\right)-\Phi(u)\right) u_{n}\left(u_{n}-u\right) \mathrm{d} x+\int_{\mathbb{R}^{3}} \Phi(u)\left(u_{n}-u\right)^{2} \mathrm{~d} x \\
& \rightarrow 0, \text { as } n \rightarrow \infty .
\end{aligned}
$$

We observe that the sequence $\left\{\Phi^{2}\left(u_{n}\right) u_{n}\right\}$ is bounded in $L^{\frac{3}{2}}\left(\mathbb{R}^{3}\right)$, since 


$$
\left|\Phi^{2}\left(u_{n}\right) u_{n}\right|_{\frac{3}{2}} \leq\left|\Phi\left(u_{n}\right)\right|_{6}^{2}\left|u_{n}\right|_{3}
$$

so that

$$
\begin{aligned}
& \left|\int_{\mathbb{R}^{3}}\left(\Phi^{2}\left(u_{n}\right) u_{n}-\Phi^{2}(u) u\right)\left(u_{n}-u\right) \mathrm{d} x\right| \\
& \leq\left|\Phi^{2}\left(u_{n}\right) u_{n}-\Phi^{2}(u) u\right|_{\frac{3}{2}}\left|u_{n}-u\right|_{3} \\
& \leq\left(\left|\Phi^{2}\left(u_{n}\right) u_{n}\right|_{\frac{3}{2}}+\left|\Phi^{2}(u) u\right|_{\frac{3}{2}}\right)\left|u_{n}-u\right|_{3} .
\end{aligned}
$$

By (16), one has

$$
\int_{\mathbb{R}^{3}}\left(\Phi^{2}\left(u_{n}\right) u_{n}-\Phi^{2}(u) u\right)\left(u_{n}-u\right) \mathrm{d} x \rightarrow 0, \text { as } n \rightarrow \infty .
$$

Therefore according to (23)-(28) and $|m|>|\omega|$, we obtain that

$$
\begin{aligned}
\left\|u_{n}-u\right\|_{H_{r}^{1}} & \leq \max \left\{1, m^{2}-\omega^{2}\right\} \int_{\mathbb{R}^{3}}\left(\left|\nabla\left(u_{n}-u\right)\right|^{2}+\left(u_{n}-u\right)^{2}\right) \mathrm{d} x \\
& \rightarrow 0, \text { as } n \rightarrow \infty .
\end{aligned}
$$

Thus $\left\{u_{n}\right\}$ has a strongly convergent subsequence in $H_{r}^{1}\left(\mathbb{R}^{3}\right)$.

Consequently, we conclude that

$$
I(u)=c, I^{\prime}(u)=0 .
$$

Next we begin to prove Theorem 2.1.

Proof. We only need to prove that $u \neq 0$. Suppose by contradiction that $u=0$, and hence $\Phi(u)=0$. Since as $n \rightarrow \infty,\left\langle I^{\prime}\left(u_{n}\right), u_{n}\right\rangle \rightarrow 0, u_{n} \rightarrow 0$ in $L_{r}^{p}\left(\mathbb{R}^{3},|x|^{-s} \mathrm{~d} x\right)\left(2<p<2^{*}(s)\right)$ and $L_{r}^{p}\left(\mathbb{R}^{3}, \mathrm{~d} x\right)\left(2<p<2^{*}\right)$. Thus we get

$$
\begin{gathered}
\int_{\mathbb{R}^{3}} u_{n}^{2} \Phi^{2}\left(u_{n}\right) \mathrm{d} x+2 \omega \int_{\mathbb{R}^{3}} \Phi\left(u_{n}\right) u_{n}^{2} \mathrm{~d} x \rightarrow 0, \\
\int_{\mathbb{R}^{3}} \frac{\left|u_{n}\right|^{p}}{|x|^{s}} \mathrm{~d} x \rightarrow 0 .
\end{gathered}
$$

We may assume

$$
\int_{\mathbb{R}^{3}}\left(\left|\nabla u_{n}\right|^{2}+\left(m^{2}-\omega^{2}\right) u_{n}^{2}\right) \mathrm{d} x=\tilde{C}\left\|u_{n}\right\|_{H_{r}^{1}}^{2}, \quad \tilde{C}>0 .
$$

Set

$$
\left\|u_{n}\right\|_{H_{r}^{1}}^{2} \rightarrow l, \quad l \geq 0,
$$

obviously, $l=0 \Leftrightarrow u_{n} \rightarrow 0$ in $H_{r}^{1}\left(\mathbb{R}^{3}\right)$. As a consequence we obtain that

$$
I\left(u_{n}\right) \rightarrow \frac{\tilde{C} l}{2}, \quad n \rightarrow \infty .
$$

According to $I\left(u_{n}\right) \rightarrow c>0$, we get

$$
c=\frac{\tilde{C} l}{2}>0,
$$

which implies that $l=0$ is impossible, i.e., which contradicts with $u=0$. Therefore, $u$ is a nontrivial solution of System (5).

This theorem is mainly based on satisfying the conditions of the Mountain 
Pass Theorem, and then there is a (PS) sequence, proving that the (PS) sequence is bounded and $u_{n}$ has a strongly convergent sub-sequence in $H_{r}^{1}\left(\mathbb{R}^{3}\right)$, so as to prove that the system of Equation (5) has at least a nontrivial solution.

\section{The Proof of Theorem 2.2}

Similarly, we also have the following lemmas.

Lemma 4.1. For every $u \in H^{1}\left(\mathbb{R}^{3}\right)$,

1) there exists a unique function $\phi=\Phi(u) \in D^{1,2}\left(\mathbb{R}^{3}\right)$ that solves the second equation of System (6);

2) if $u$ is radially symmetric, then $\Phi(u)$ is radial too;

3) $\Phi(u)(x) \leq 0$, moreover, $\Phi(u)(x) \geq-\omega$, if $u(x) \neq 0$ and $\omega>0$.

Lemma 4.2. The map $\Phi$ is $C^{1}$ and $\tilde{G}_{\phi}=\left\{(u, \phi) \in H^{1}\left(\mathbb{R}^{3}\right) \times D^{1,2}\left(\mathbb{R}^{3}\right) \mid \tilde{F}_{\phi}^{\prime}(u, \phi)=0\right\}$.

Likewise, define $J(u)=\tilde{F}(u, \phi)$.

Lemma 4.3. The pair $(u, \phi)$ is a weak solution of System (6) if and only if it is a critical point of J in $H^{1}\left(\mathbb{R}^{3}\right) \times D^{1,2}\left(\mathbb{R}^{3}\right)$.

Lemma 4.4 For $u \in H^{1}\left(\mathbb{R}^{3}\right)$, if $|m|>|\omega|$, then there exist some constants $\rho_{2}, \alpha_{2}, \tilde{m}>0$ such that $\left.J(u)\right|_{\|\mu\|_{H^{1}=\rho_{2}}} \geq \alpha_{2}$ for all $\mu$ satisfying $0<\mu<\tilde{m}$.

Proof. From (8), one obtains

$$
\begin{aligned}
J(u)= & \frac{1}{2} \int_{\mathbb{R}^{3}}\left(|\nabla u|^{2}+\left(m^{2}-\omega^{2}\right) u^{2}\right) \mathrm{d} x-\frac{1}{2} \int_{\mathbb{R}^{3}}|\nabla \phi|^{2} \mathrm{~d} x-\int_{\mathbb{R}^{3}} \omega \phi u^{2} \mathrm{~d} x \\
& -\frac{1}{2} \int_{\mathbb{R}^{3}} \phi^{2} u^{2} \mathrm{~d} x-\frac{\mu}{q} \int_{\mathbb{R}^{3}} \frac{|u|^{q}}{|x|^{s}} \mathrm{~d} x-\frac{1}{p} \int_{\mathbb{R}^{3}} \frac{|u|^{p}}{|x|^{s}} \mathrm{~d} x-\frac{1}{2^{*}(s)} \int_{\mathbb{R}^{3}} \frac{|u|^{2^{*}(s)}}{|x|^{s}} \mathrm{~d} x .
\end{aligned}
$$

Substituting (9) into (29), we have

$$
\begin{aligned}
J(u)= & \frac{1}{2} \int_{\mathbb{R}^{3}}\left(|\nabla u|^{2}+\left(m^{2}-\omega^{2}\right) u^{2}\right) \mathrm{d} x+\frac{1}{2} \int_{\mathbb{R}^{3}}|\nabla \phi|^{2} \mathrm{~d} x+\frac{1}{2} \int_{\mathbb{R}^{3}} \phi^{2} u^{2} \mathrm{~d} x \\
& -\frac{\mu}{q} \int_{\mathbb{R}^{3}} \frac{|u|^{q}}{|x|^{s}} \mathrm{~d} x-\frac{1}{p} \int_{\mathbb{R}^{3}} \frac{|u|^{p}}{|x|^{s}} \mathrm{~d} x-\frac{1}{2^{*}(s)} \int_{\mathbb{R}^{3}} \frac{|u|^{2^{*}(s)}}{|x|^{s}} \mathrm{~d} x \\
\geq & \frac{1}{2} \int_{\mathbb{R}^{3}}\left(|\nabla u|^{2}+\left(m^{2}-\omega^{2}\right) u^{2}\right) \mathrm{d} x-\frac{\mu}{q} \int_{\mathbb{R}^{3}} \frac{|u|^{q}}{|x|^{s}} \mathrm{~d} x \\
& -\frac{1}{p} \int_{\mathbb{R}^{3}} \frac{|u|^{p}}{|x|^{s}} \mathrm{~d} x-\frac{1}{2^{*}(s)} \int_{\mathbb{R}^{3}} \frac{|u|^{2^{*}(s)}}{|x|^{s}} \mathrm{~d} x \\
\geq & \frac{\min \left\{1, m^{2}-\omega^{2}\right\}}{2}\|u\|_{H^{1}}^{2}-\frac{C_{11} \mu}{q}\|u\|_{H^{1}}^{q}-\frac{C_{12}}{p}\|u\|_{H^{1}}^{p}-\frac{1}{2^{*}(s)} S_{s}^{-2^{2^{*}(s)}} \\
= & \|u\|_{H^{1}}^{q}\left(\frac{\min \left\{1, m^{2}-\omega^{2}\right\}}{2}\|u\|_{H^{1}}^{2-q}-\frac{C_{12}}{p}\|u\|_{H^{1}}^{p-q}-\frac{1}{2^{*}(s)} S_{s}^{-\frac{2^{*}(s)}{2}}\|u\|_{H^{1}}^{2^{*}(s)-q}-\frac{C_{11} \mu}{q}\right) . \\
\text { Set } & h_{1}(\rho)=\frac{\min \left\{1, m^{2}-\omega^{2}\right\}}{2} \rho^{2-q}-\frac{C_{12}}{p} \rho^{p-q}-\frac{1}{2^{*}(s)} S_{s}^{-\frac{2^{*}(s)}{2}} \rho^{2^{*}(s)-q}, \rho \geq 0 .
\end{aligned}
$$

Since 


$$
\begin{aligned}
h_{1}^{\prime}(\rho)= & \frac{\left(\min \left\{1, m^{2}-\omega^{2}\right\}\right)(2-q)}{2} \rho^{1-q}-\frac{C_{12}(p-q)}{p} \rho^{p-q-1} \\
& -\frac{2^{*}(s)-q}{2^{*}(s)} S_{s}^{-2^{*}(s)} \rho^{2^{*}(s)-q-1} \\
= & \rho^{1-q}\left(\frac{\left(\min \left\{1, m^{2}-\omega^{2}\right\}\right)(2-q)}{2}-\frac{C_{12}(p-q)}{p} \rho^{p-2}\right. \\
& \left.-\frac{2^{*}(s)-q}{2^{*}(s)} S_{s}^{-\frac{2^{*}(s)}{2}} \rho^{2^{*}(s)-2}\right) .
\end{aligned}
$$

Evidently, when $\rho \geq 0$ is small enough, $h_{1}^{\prime}(\rho)$ is greater than 0 , and $h_{1}(\rho)$ increases monotonically. When $\rho \geq 0$ is large enough, $h_{1}^{\prime}(\rho)$ is less than 0 , and $h_{1}(\rho)$ decreases monotonically. Therefore there exists a maximum point $\tilde{\xi}$ such that

$$
h_{1}^{\prime}(\tilde{\xi})=0 .
$$

Obviously, we observe that $\tilde{\xi}=0$ or $\tilde{\xi}>0$, while $\tilde{\xi}=0$ is impossible. Then $\tilde{\xi}>0$, next we obtain $\max _{\rho \geq 0} h_{1}(\rho)=h_{1}(\tilde{\xi})>0$. Choosing that $\|u\|_{H^{1}}=\tilde{\xi}=\rho_{2}$, we deduce, for all $\mu$ satisfying $0<\mu<\frac{q h_{1}\left(\rho_{2}\right)}{2 C_{11}}=\tilde{m}$,

$$
\left.J(u)\right|_{\|u\|_{H^{1}}=\rho_{2}} \geq \rho_{2}^{q} \cdot\left(h_{1}\left(\rho_{2}\right)-\frac{C_{11} \mu}{q}\right) \geq \frac{\rho_{2}^{q} \cdot h_{1}\left(\rho_{2}\right)}{2}=\alpha_{2}>0
$$

and the proof is completed.

Lemma 4.5. Under the assumptions of Theorem 2.2, there exists a function $\eta_{2} \in H^{1}\left(\mathbb{R}^{3}\right)$ with $\left\|\eta_{2}\right\|_{H^{1}}>\rho_{2}$ such that $J\left(\eta_{2}\right)<0$.

Similarly, a standard tool is to restrict ourselves to radial functions, namely $H_{r}^{1}\left(\mathbb{R}^{3}\right)=\left\{u \in H^{1}\left(\mathbb{R}^{3}\right) \mid u(x)=u(|x|)\right\}$ and $D_{r}^{1,2}\left(\mathbb{R}^{3}\right)=\left\{u \in D^{1,2}\left(\mathbb{R}^{3}\right) \mid u(x)=u(|x|)\right\}$ compactly embedded in $L_{r}^{p}\left(\mathbb{R}^{3},|x|^{-s} \mathrm{~d} x\right)$ for $2<p<2^{*}(s)$ and $L_{r}^{p}\left(\mathbb{R}^{3}, \mathrm{~d} x\right)$ for $2<p<2^{*}=\frac{2 N}{N-2}=6$. Moreover, one sees that if a critical point $u \in H_{r}^{1}\left(\mathbb{R}^{3}\right)$ for the functional $\left.J\right|_{H_{r}^{1}\left(\mathbb{R}^{3}\right)}$ is also a critical point of $J$.

Lemma 4.6. Under the assumptions of Theorem 2.2, if $\left\{u_{n}\right\} \in H_{r}^{1}\left(\mathbb{R}^{3}\right)$ is a bounded Palais-Smale sequence of $J$, then $\left\{u_{n}\right\}$ has a strongly convergent subsequence in $H_{r}^{1}\left(\mathbb{R}^{3}\right)$.

Proof. Consider a sequence $\left\{u_{n}\right\}$ in $H_{r}^{1}\left(\mathbb{R}^{3}\right)$, which satisfies $J\left(u_{n}\right) \rightarrow c$, $J^{\prime}\left(u_{n}\right) \rightarrow 0$, and $\sup \left\|u_{n}\right\|_{H_{r}^{1}}<+\infty$. Going if necessary to a subsequence, we assume

$$
u_{n} \rightarrow u \text { in } H_{r}^{1}\left(\mathbb{R}^{3}\right) .
$$

Since the embedding $H_{r}^{1}\left(\mathbb{R}^{3}\right) \hookrightarrow L_{r}^{p}\left(\mathbb{R}^{3},|x|^{-s} \mathrm{~d} x\right)$ is compact for any 
$p \in\left(2,2^{*}(s)\right)$, we have

$$
u_{n} \rightarrow u \text { in } L_{r}^{p}\left(\mathbb{R}^{3},|x|^{-s} \mathrm{~d} x\right) .
$$

Moreover, likewise, for $p \in\left(2,2^{*}\right)$, we also get

$$
u_{n} \rightarrow u \text { in } L_{r}^{p}\left(\mathbb{R}^{3}, \mathrm{~d} x\right)
$$

According to

$$
\begin{aligned}
J^{\prime}(u) v= & \int_{\mathbb{R}^{3}}\left(\nabla u \cdot \nabla v+\left[m^{2}-(\omega+\phi)^{2}\right] u v\right) \mathrm{d} x-\mu \int_{\mathbb{R}^{3}} \frac{|u|^{q-2}}{|x|^{s}} u v \mathrm{~d} x \\
& -\int_{\mathbb{R}^{3}} \frac{|u|^{p-2}}{|x|^{s}} u v \mathrm{~d} x-\int_{\mathbb{R}^{3}} \frac{|u|^{2^{*}(s)-2}}{|x|^{s}} u v \mathrm{~d} x,
\end{aligned}
$$

one obtains

$$
\begin{aligned}
& J^{\prime}\left(u_{n}\right)\left(u_{n}-u\right) \\
& =\int_{\mathbb{R}^{3}}\left(\nabla u_{n} \cdot \nabla\left(u_{n}-u\right)+\left[m^{2}-\left(\omega+\Phi\left(u_{n}\right)\right)^{2}\right] u_{n}\left(u_{n}-u\right)\right) \mathrm{d} x \\
& -\mu \int_{\mathbb{R}^{3}} \frac{\left|u_{n}\right|^{q-2}}{|x|^{s}} u_{n}\left(u_{n}-u\right) \mathrm{d} x-\int_{\mathbb{R}^{3}} \frac{\left|u_{n}\right|^{p-2}}{|x|^{s}} u_{n}\left(u_{n}-u\right) \mathrm{d} x \\
& -\int_{\mathbb{R}^{3}} \frac{\left|u_{n}\right|^{2^{*}(s)-2}}{|x|^{s}} u_{n}\left(u_{n}-u\right) \mathrm{d} x .
\end{aligned}
$$

Similarly, one gets

$$
\begin{aligned}
& J^{\prime}(u)\left(u_{n}-u\right) \\
& =\int_{\mathbb{R}^{3}}\left(\nabla u \cdot \nabla\left(u_{n}-u\right)+\left[m^{2}-(\omega+\Phi(u))^{2}\right] u\left(u_{n}-u\right)\right) \mathrm{d} x \\
& -\mu \int_{\mathbb{R}^{3}} \frac{|u|^{q-2}}{|x|^{s}} u\left(u_{n}-u\right) \mathrm{d} x-\int_{\mathbb{R}^{3}} \frac{|u|^{p-2}}{|x|^{s}} u\left(u_{n}-u\right) \mathrm{d} x \\
& -\int_{\mathbb{R}^{3}} \frac{|u|^{2^{*}(s)-2}}{|x|^{s}} u\left(u_{n}-u\right) \mathrm{d} x .
\end{aligned}
$$

By (32), we easily get that

$$
\begin{aligned}
& \int_{\mathbb{R}^{3}}\left(\left|\nabla\left(u_{n}-u\right)\right|^{2}+\left(m^{2}-\omega^{2}\right)\left(u_{n}-u\right)^{2}\right) \mathrm{d} x \\
& =\left\langle J^{\prime}\left(u_{n}\right)-J^{\prime}(u), u_{n}-u\right\rangle+\mu \int_{\mathbb{R}^{3}}\left(\frac{\left|u_{n}\right|^{q-2}}{|x|^{s}} u_{n}-\frac{|u|^{q-2}}{|x|^{s}} u\right)\left(u_{n}-u\right) \mathrm{d} x \\
& +\int_{\mathbb{R}^{3}}\left(\frac{\left|u_{n}\right|^{p-2}}{|x|^{s}} u_{n}-\frac{|u|^{p-2}}{|x|^{s}} u\right)\left(u_{n}-u\right) \mathrm{d} x \\
& +2 \omega \int_{\mathbb{R}^{3}}\left(\Phi\left(u_{n}\right) u_{n}-\Phi(u) u\right)\left(u_{n}-u\right) \mathrm{d} x \\
& +\int_{\mathbb{R}^{3}}\left(\frac{\left|u_{n}\right|^{2^{*}(s)-2}}{|x|^{s}} u_{n}-\frac{|u|^{2^{*}(s)-2}}{|x|^{s}} u\right)\left(u_{n}-u\right) \mathrm{d} x \\
& +\int_{\mathbb{R}^{3}}\left(\Phi^{2}\left(u_{n}\right) u_{n}-\Phi^{2}(u) u\right)\left(u_{n}-u\right) \mathrm{d} x .
\end{aligned}
$$

Then we use similar method in the proof of Lemma 3.8, we obtain that $\left\{u_{n}\right\}$ 
has a strongly convergent subsequence in $H_{r}^{1}\left(\mathbb{R}^{3}\right)$.

Next we begin to prove Theorem 2.2.

Proof. The proof is divided into two steps.

Step 1. There exists $u_{1} \in H_{r}^{1}\left(\mathbb{R}^{3}\right)$ such that $J^{\prime}\left(u_{1}\right)=0$ and $J\left(u_{1}\right)<0$.

We choose a function $v \in H_{r}^{1}\left(\mathbb{R}^{3}\right)$. Since $\Phi(u) \geq-\omega$, one has

$$
\begin{aligned}
J(t v)= & \frac{t^{2}}{2} \int_{\mathbb{R}^{3}}\left(|\nabla v|^{2}+\left(m^{2}-\omega^{2}\right) v^{2}\right) \mathrm{d} x-t^{2} \int_{\mathbb{R}^{3}} \omega \Phi(t v) v^{2} \mathrm{~d} x \\
& -\frac{t^{2}}{2} \int_{\mathbb{R}^{3}} \Phi^{2}(t v) v^{2} \mathrm{~d} x-\frac{1}{2} \int_{\mathbb{R}^{3}}|\nabla \Phi(t v)|^{2} \mathrm{~d} x \\
& -\frac{\mu t^{q}}{q} \int_{\mathbb{R}^{3}} \frac{|v|^{q}}{|x|^{s}} \mathrm{~d} x-\frac{t^{p}}{p} \int_{\mathbb{R}^{3}} \frac{|v|^{p}}{|x|^{s}} \mathrm{~d} x-\frac{t^{2^{*}(s)}}{2^{*}(s)} \int_{\mathbb{R}^{3}} \frac{|v|^{2^{*}(s)}}{|x|^{s}} \mathrm{~d} x \\
\leq & \frac{t^{2}}{2} \int_{\mathbb{R}^{3}}\left(|\nabla v|^{2}+\left(m^{2}-\omega^{2}\right) v^{2}+2 \omega^{2} v^{2}\right) \mathrm{d} x-\frac{\mu t^{q}}{q} \int_{\mathbb{R}^{3}} \frac{|v|^{q}}{|x|^{s}} \mathrm{~d} x \\
< & 0
\end{aligned}
$$

for $t>0$ small enough. Thus we have $c_{1}=\inf \left\{J(u): u \in \bar{B}_{\rho_{2}}\right\}<0$, where $\rho_{2}>0$ is given by Lemma 4.4, $B_{\rho_{2}}=\left\{u \in H_{r}^{1}\left(\mathbb{R}^{3}\right):\|u\|_{H_{r}^{1}}<\rho_{2}\right\}$. By the Ekeland's variational principle [17], Let $\varepsilon=\frac{1}{n}>0, \delta=\left\|\zeta-u_{n}\right\|_{H_{r}^{1}}>0$ for all $\zeta \in \bar{B}_{\rho_{2}}$, then there exists a sequence $\left\{u_{n}\right\} \subset \bar{B}_{\rho_{2}}$ such that

$$
c_{1} \leq J\left(u_{n}\right) \leq c_{1}+\varepsilon
$$

and

$$
c_{1}+\frac{\varepsilon}{\delta}\left\|\zeta-u_{n}\right\|_{H_{r}^{1}} \leq J(\zeta)+\frac{\varepsilon}{\delta}\left\|\zeta-u_{n}\right\|_{H_{r}^{1}} .
$$

Then we obtain that

$$
J(\zeta) \geq J\left(u_{n}\right)-\frac{\varepsilon}{\delta}\left\|\zeta-u_{n}\right\|_{H_{r}^{1}}
$$

Obviously, in view of Lemma 4.4, $u_{n} \in B_{\rho_{2}}$, for $n$ large enough. Thus for any $\phi \in H_{r}^{1}\left(\mathbb{R}^{3}\right)$ with $\|\phi\|_{H_{r}^{1}}=1$, we can take $t>0$ such that $\left(u_{n}+t \phi\right) \in \bar{B}_{\rho_{2}}$ for $n$ large enough (see [18]). Then we have

$$
\frac{J\left(u_{n}+t \phi\right)-J\left(u_{n}\right)}{t} \geq-\varepsilon .
$$

Letting $t \rightarrow 0$, we get

$$
\left\langle J^{\prime}\left(u_{n}\right), \phi\right\rangle \geq-\varepsilon
$$

We replace $\phi$ by $-\phi$ in the above inequality, then it follows that

$$
\left\langle J^{\prime}\left(u_{n}\right),-\phi\right\rangle \geq-\varepsilon,
$$

i.e.,

$$
\left\langle J^{\prime}\left(u_{n}\right), \phi\right\rangle \leq \varepsilon .
$$


Thus one obtains

$$
\left|\left\langle J^{\prime}\left(u_{n}\right), \phi\right\rangle\right| \leq \varepsilon
$$

which implies $J^{\prime}\left(u_{n}\right) \rightarrow 0$ as $n \rightarrow \infty$.

Hence we conclude that $\left\{u_{n}\right\}$ is bounded $P S$ sequence of $J$ for $c_{1}$. Therefore, by Lemma 4.6 , we get that there exists a function $u_{1} \in H_{r}^{1}\left(\mathbb{R}^{3}\right)$ such that $J^{\prime}\left(u_{1}\right)=0$ and $J\left(u_{1}\right)=c_{1}<0$.

Step 2. There exists $u_{2} \in H_{r}^{1}\left(\mathbb{R}^{3}\right)$ such that $J^{\prime}\left(u_{2}\right)=0$ and $J\left(u_{2}\right)>0$.

From Lemma 4.4, Lemma 4.5 and the Mountain Pass Theorem [19], there is a sequence $\left\{u_{n}\right\} \subset H_{r}^{1}\left(\mathbb{R}^{3}\right)$ such that

$$
J\left(u_{n}\right) \rightarrow c_{2}=\inf _{\gamma \in \Gamma} \max _{t \in[0,1]} J(\gamma(t)) \geq \alpha_{2}>0
$$

and

$$
J^{\prime}\left(u_{n}\right) \rightarrow 0
$$

where $\Gamma=\left\{\gamma \in C\left([0,1], H_{r}^{1}\left(\mathbb{R}^{3}\right)\right): \gamma(0)=0, \gamma(1)=\eta_{2}\right\}$. From Lemma 4.6, we only need to prove that $\left\{u_{n}\right\}$ is bounded in $H_{r}^{1}\left(\mathbb{R}^{3}\right)$.

Case 1): $4 \leq p<2^{*}(s)$. From (8) and (9), one has

$$
\begin{aligned}
J(u)= & \frac{1}{2} \int_{\mathbb{R}^{3}}\left(|\nabla u|^{2}+\left(m^{2}-\omega^{2}\right) u^{2}\right) \mathrm{d} x+\frac{1}{2} \int_{\mathbb{R}^{3}} \Phi^{2}(u) u^{2} \mathrm{~d} x+\frac{1}{2} \int_{\mathbb{R}^{3}}|\nabla \Phi(u)|^{2} \mathrm{~d} x \\
& -\frac{\mu}{q} \int_{\mathbb{R}^{3}} \frac{|u|^{q}}{|x|^{s}} \mathrm{~d} x-\frac{1}{p} \int_{\mathbb{R}^{3}} \frac{|u|^{p}}{|x|^{s}} \mathrm{~d} x-\frac{1}{2^{*}(s)} \int_{\mathbb{R}^{3}} \frac{|u|^{2^{*}(s)}}{|x|^{s}} \mathrm{~d} x .
\end{aligned}
$$

Then by (32), one obtains

$$
\begin{aligned}
& \left(c_{2}+1\right)+o(1)\left\|u_{n}\right\|_{H_{r}^{1}} \\
& \geq J\left(u_{n}\right)-\frac{1}{p}\left\langle J^{\prime}\left(u_{n}\right), u_{n}\right\rangle \\
& =\left(\frac{1}{2}-\frac{1}{p}\right) \int_{\mathbb{R}^{3}}\left(\left|\nabla u_{n}\right|^{2}+\left(m^{2}-\omega^{2}\right) u_{n}^{2}\right) \mathrm{d} x+\left(\frac{1}{2}+\frac{1}{p}\right) \int_{\mathbb{R}^{3}} \Phi^{2}\left(u_{n}\right) u_{n}^{2} \mathrm{~d} x \\
& +\frac{1}{2} \int_{\mathbb{R}^{3}}\left|\nabla \Phi\left(u_{n}\right)\right|^{2} \mathrm{~d} x+\mu\left(\frac{1}{p}-\frac{1}{q}\right) \int_{\mathbb{R}^{3}} \frac{\left|u_{n}\right|^{q}}{|x|^{s}} \mathrm{~d} x \\
& +\frac{2}{p} \int_{\mathbb{R}^{3}} \omega \Phi\left(u_{n}\right) u_{n}^{2} \mathrm{~d} x+\left(\frac{1}{p}-\frac{1}{2^{*}(s)}\right) \int_{\mathbb{R}^{3}} \frac{\left|u_{n}\right|^{2^{*}(s)}}{|x|^{s}} \mathrm{~d} x \\
& =\left(\frac{1}{2}-\frac{1}{p}\right) \int_{\mathbb{R}^{3}}\left(\left|\nabla u_{n}\right|^{2}+\left(m^{2}-\omega^{2}\right) u_{n}^{2}\right) \mathrm{d} x+\left(\frac{1}{2}-\frac{1}{p}\right) \int_{\mathbb{R}^{3}} \Phi^{2}\left(u_{n}\right) u_{n}^{2} \mathrm{~d} x \\
& +\left(\frac{1}{2}-\frac{2}{p}\right) \int_{\mathbb{R}^{3}}\left|\nabla \Phi\left(u_{n}\right)\right|^{2} \mathrm{~d} x+\mu\left(\frac{1}{p}-\frac{1}{q}\right) \int_{\mathbb{R}^{3}} \frac{\left|u_{n}\right|^{q}}{|x|^{s}} \mathrm{~d} x \\
& +\left(\frac{1}{p}-\frac{1}{2^{*}(s)}\right) \int_{\mathbb{R}^{3}} \frac{\left|u_{n}\right|^{2^{*}(s)}}{|x|^{s}} \mathrm{~d} x \\
& \geq C_{13}\left\|u_{n}\right\|_{H_{r}^{1}}^{2}+\mu\left(\frac{1}{p}-\frac{1}{q}\right) C_{14}\left\|u_{n}\right\|_{H_{r}^{1}}^{q}
\end{aligned}
$$


for $n$ large enough. Therefore, it follows that $\left\{u_{n}\right\}$ is bounded in $H_{r}^{1}\left(\mathbb{R}^{3}\right)$.

Case 2): $2<p<4$. From (8) and (9), one has

$$
\begin{aligned}
J(u)= & \frac{1}{2} \int_{\mathbb{R}^{3}}\left(|\nabla u|^{2}+\left(m^{2}-\omega^{2}\right) u^{2}\right) \mathrm{d} x-\frac{1}{2} \int_{\mathbb{R}^{3}} \omega \Phi(u) u^{2} \mathrm{~d} x-\frac{\mu}{q} \int_{\mathbb{R}^{3}} \frac{|u|^{q}}{|x|^{s}} \mathrm{~d} x \\
& -\frac{1}{p} \int_{\mathbb{R}^{3}} \frac{|u|^{p}}{|x|^{s}} \mathrm{~d} x-\frac{1}{2^{*}(s)} \int_{\mathbb{R}^{3}} \frac{|u|^{2^{*}(s)}}{|x|^{s}} \mathrm{~d} x .
\end{aligned}
$$

Then by (32) and $\left(\frac{p}{2}-1\right) m^{2}>\omega^{2}$ and $\Phi\left(u_{n}\right) \geq-\omega, 1<q<2$, we get

$$
\begin{aligned}
& p\left(c_{2}+1\right)+\left\|u_{n}\right\|_{H_{r}^{1}} \\
\geq & p J\left(u_{n}\right)-\left\langle J^{\prime}\left(u_{n}\right), u_{n}\right\rangle \\
= & \left(\frac{p}{2}-1\right) \int_{\mathbb{R}^{3}}\left(\left|\nabla u_{n}\right|^{2}+\left(m^{2}-\omega^{2}\right) u_{n}^{2}\right) \mathrm{d} x-\left(\frac{p}{2}-2\right) \int_{\mathbb{R}^{3}} \omega \Phi\left(u_{n}\right) u_{n}^{2} \mathrm{~d} x \\
& +\int_{\mathbb{R}^{3}} \Phi^{2}\left(u_{n}\right) u_{n}^{2} \mathrm{~d} x+\mu\left(1-\frac{p}{q}\right) \int_{\mathbb{R}^{3}} \frac{\left|u_{n}\right|^{q}}{|x|^{s}} \mathrm{~d} x+\left(1-\frac{p}{2^{*}(s)}\right) \int_{\mathbb{R}^{3}} \frac{\left|u_{n}\right|^{2^{*}(s)}}{|x|^{s}} \mathrm{~d} x \\
\geq & \left(\frac{p}{2}-1\right) \int_{\mathbb{R}^{3}}\left|\nabla u_{n}\right|^{2} \mathrm{~d} x+\int_{\mathbb{R}^{3}}\left[\left(\frac{p}{2}-1\right)\left(m^{2}-\omega^{2}\right)+\left(\frac{p}{2}-2\right) \omega^{2}\right] u_{n}^{2} \mathrm{~d} x \\
& +\mu\left(1-\frac{p}{q}\right) \int_{\mathbb{R}^{3}} \frac{\left|u_{n}\right|^{q}}{|x|^{s}} \mathrm{~d} x \\
\geq & C_{15}\left\|u_{n}\right\|_{H_{r}^{1}}^{2}+\mu\left(1-\frac{p}{q}\right) C_{16}\left\|u_{n}\right\|_{H_{r}^{1}}^{q}
\end{aligned}
$$

for $n$ large enough. It follows that $\left\{u_{n}\right\}$ is bounded in $H_{r}^{1}\left(\mathbb{R}^{3}\right)$.

This theorem is mainly based on the Mountain Pass Theorem and the Ekeland variational principle to prove that the System (6) has at least two different solutions.

Remark 4.1. Here a simple infimum definition is given as follows. Suppose $S$ is a set of numbers in $\mathbb{R}$. If the number $\xi_{0}$ satisfies.

1) $\forall x \in S, x \geq \xi_{0}$, that is, $\xi_{0}$ is a lower bound of $S$.

2) $\forall \beta_{0}>\xi_{0}$, there exists a $x_{0} \in S$ that satisfies $x_{0}<\beta_{0}$, that is, $\xi_{0}$ is maximum lower bound of $S$, then the number $\xi_{0}$ is the infimum of the number set. Referred to as: $\xi_{0}=\inf S$.

\section{Conflicts of Interest}

The author declares no conflicts of interest.

\section{References}

[1] Bertoin, J. (1996) Lévy Processes. Volume 121 of Cambridge Tracts in Mathematics. Cambridge University Press, Cambridge.

[2] Bucur, C. and Valdinoci, E. (2016) Nonlocal Diffusion and Applications. Volume 20 of Lecture Notes of the Unione Matematica Italiana. Springer, Cham. https://doi.org/10.1007/978-3-319-28739-3

[3] Bhakta, M. (2015) Semilinear Elliptic Equation with Biharmonic Operator and Mul- 
tiple Critical Nonlinearities. Advanced Nonlinear Studies, 15, 835-848. https://doi.org/10.1515/ans-2015-0405

[4] Filippucci, R., Pucci, P. and Robert, F. (2009) On a p-Laplace Equation with Multiple Critical Nonlinearities. Journal de Mathématiques Pures et Appliquées, 91, 156-177. https://doi.org/10.1016/j.matpur.2008.09.008

[5] Ghoussoub, N. and Robert, F. (2006) Concentration Estimates for Emden-Fowler Equations with Boundary Singularities and Critical Growth. International Mathematics Research Papers, 2006, Article ID: 21867.

[6] Brézis, H. and Nirenberg, L. (1983) Positive Solutions of Nonlinear Elliptic Equations Involving Critical Sobolev Exponents. Communications on Pure and Applied Mathematics, 36, 437-477. https://doi.org/10.1002/cpa.3160360405

[7] Jannelli, E. (1999) The Role Played by Space Dimension in Elliptic Critical Problems. Journal of Differential Equations, 156, 407-426.

https://doi.org/10.1006/jdeq.1998.3589

[8] Kang, D. and Peng, S. (2004) Existence of Solutions for Elliptic Equations with Critical Sobolev-Hardy Exponents. Nonlinear Analysis, 56, 1151-1164. https://doi.org/10.1016/j.na.2003.11.008

[9] Kang, D. and Peng, S. (2005) Solutions for Semilinear Elliptic Problems with Critical Sobolev-Hardy Exponents and Hardy Potential. Applied Mathematics Letters, 18, 1094-1100. https://doi.org/10.1016/j.aml.2004.09.016

[10] Kang, D. and Peng, S. (2004) Sign-Changing Solutions for Elliptic Problems with Critical Sobolev-Hardy Exponents. Journal of Mathematical Analysis and Applications, 291, 488-499. https://doi.org/10.1016/j.jmaa.2003.11.012

[11] Benci, V. and Fortunato, D. (2002) Solitary Waves of the Nonlinear Klein-Gordon Equation Coupled with the Maxwell Equations. Reviews in Mathematical Physics, 14, 409-420. https://doi.org/10.1142/S0129055X02001168

[12] Berestycki, H. and Lions, P.-L. (1983) Nonlinear Scalar Field Equations, I-Existence of a Ground State. Archive for Rational Mechanics and Analysis, 82, 313-345. https://doi.org/10.1007/BF00250555

[13] Cassani, D. (2004) Existence and Non-Existence of Solitary Waves for the Critical Klein-Gordon Equation Coupled with Maxwell's Equations. Nonlinear Analysis, 58, 733-747. https://doi.org/10.1016/j.na.2003.05.001

[14] d'Avenia, P. and Pisani, L. (2002) Nonlinear Klein-Gordon Equations Coupled with Born-Infeld Type Equations. Electronic Journal of Differential Equations, 2002, 1-13.

[15] Mugnai, D. (2004) Coupled Klein-Gordon and Born-Infeld-Type Equations: Looking for Solitary Waves. Proceedings of the Royal Society A: Mathematical, Physical and Engineering Sciences, 460, 1519-1527. https://doi.org/10.1098/rspa.2003.1267

[16] Yu, Y. (2010) Solitary Waves for Nonlinear Klein-Gordon Equations Coupled with Born-Infeld Theory. Annales de P Institut Henri Poincaré, Analyse Non Linéaire, 27, 351-376. https://doi.org/10.1016/j.anihpc.2009.11.001

[17] Ekeland, I. (1974) On the Variational Principle. Journal of Mathematical Analysis and Applications, 47, 324-353. https://doi.org/10.1016/0022-247X(74)90025-0

[18] Chen, S.-J. and Song, S.-Z. (2017) The Existence of Multiple Solutions for the Klein-Gordon Equation with Concave and Convex Nonlinearities Coupled with Born-Infeld Theory on $\mathrm{R}^{3}$. Nonlinear Analysis. Real World Applications, 38, 78-95. https://doi.org/10.1016/j.nonrwa.2017.04.008

[19] Willem, M. (1996) Minimax Theorems (Progress in Nonlinear Differential Equations and Their Applications). Vol. 24, Birkhäuser Boston, Inc., Boston. 